\title{
Passage organization and imposed learning strategies in comprehension and recall of connected discourse:
}

\author{
FRANCIS J. DI VESTA, CHARLES B. SCHULTZ† \\ and \\ TIMOTHY R. DANGEL \\ Pennsylvania State University, University Park, Pennsylvania 16802
}

\begin{abstract}
Ninety-nine Ss were assigned randomly to learn a passage comprised of six paragraphs in which the statements were organized by concept name, or concept attribute, or in which the statements were scrambled. Each complete passage contained the same 36 statements. These treatments were orthogonally crossed with instructions to employ a name clustering strategy, an attribute clustering strategy, or a subjectively determined organizing strategy. Three learning trials were administered, each of which was followed by free recall. Passages organized by concept name were found to result in greater recall than passages organized by attributes. The name clustering strategy was more dominant than the attribute clustering strategy. Incongruence between passage organization and advocated clustering strategy resulted in greater recall than did congruency. Implications of these results for cognitive processing of information are discussed.
\end{abstract}

The organization of passages in connected discourse by concept name or concept attribute differentially influences learning strategies. Passages organized by name or attribute facilitate recall more than passages without organizational patterns; clustering during recall is influenced by passage organization; and the concept-name clustering strategy during recall is more dominant than the concept-attribute strategy (Frase, 1969; Schultz \& Di Vesta, 1972).

An interesting implication from a study by Schultz and Di Vesta (1972) was that the concept-name and concept-attribute clustering strategies were possibly adopted at different rates. Thus, the concept-name clustering strategy was used from the time of the first encounter with passages organized by name, but the concept-attribute strategy was adopted only after several exposures to passages organized by attribute. The evidence available at that time implied that, when the dominant clustering strategy (organization by concept name) was congruent with the passage organization, it was immediately adopted. On the other hand, when the subordinate strategy (organization by concept attribute) was congruent with the passage organization, it was gradually adopted, perhaps because of interference from the dominant strategy. Furthermore, recall was impaired and errors were more frequent during the first trial of learning a passage organized by attribute. Presumably, it is on this trial that reliance on the incongruent concept-name strategy is greatest. This finding implied that a subjective clustering strategy might be a

\footnotetext{
*The research reported in this paper was supported in part by the Advanced Research Projects Agency (ARPA Order No. 1269) through the Office of Naval Research under Contract ONR Nonr N00014-67-A-0385-0006. The authors are indebted to Dr. Paul Games for his consultation regarding the statistical analyses employed in this study.

$\div$ Now at Trinity College. Hartford. Connecticut.
}

decremental factor in the learning and retention of textual material when it is incongruent with the organizational structure of the passage.

On the basis of the above reasoning, it was hypothesized that, through instructions to the $S$, the imposition of a clustering strategy congruent with the passage organization would result in greater recall and fewer errors than when the imposed clustering strategy was incongruent with the passage organization. This hypothesis was examined via a design; in which learners were required to adopt elther the domınant strategy; the subordinate strategy, or, in a third treatment, were free to adopt any strategy they chose to use.

\section{METHOD}

Design

The Ss were given three trials to learn a passage that described six imaginary nations. Each trial was comprised of a brief study period followed by a free-recall test. Following the third trial. the Ss completed a task intended to prevent rehearsal of the passage before the administration of a fourth free-recall test. The number of correct responses and clustering ratios were obtained on each trial of the free-recall test.

Three levels of passage organization [concept name (CNP), concept attribute (CAP), and random sentence sequence (ROP)] were orthogonally crossed with three sets of instructions for grouping the statements in the passage [name grouping (CNI), attribute grouping (CAI), and no specific grouping (NOI) instructions]. The data were analyzed via separate 3 by 3 by 4 mixed analyses of variance (with repeated measures on the last factor, i.e., free-recall trials) for each dependent variable (see the section on scoring below).

\section{Subjects}

The Ss were 99 undergraduate students enrolled in an introductory educational psychology course at The Pennsylvania State Lniversity. Students were awarded standard score points toward their grade for participation in the experiment. The experimental sessions were conducted with groups comprised of 
nine Ss. Each $S$ within a group was assigned randomly to a different experimental condition $(N=11)$.

\section{Experimental Materials}

The study passage was similar to that used in an earlier experiment (Schultz \& Di Vesta. 1972). It consisted of 36 statements describing six imaginary nations (concepts) called Atweena, Brontus, Egrama. Nurovia. Bismania, and Galbion. The geography. government. mood, technology, population growth. and birth rate were the attributes described for each nation. The same 36 statements were used to construct three passages with different organizational patterns. In one passage organization of these statements, each of six paragraphs contained six sentences about one of the nations (Condition CNP). In a second organizational pattern. each paragraph was comprised of those statements describing the same attribute for each of the six nations (Condition CAP). A third passage contained six paragraphs, each of which was composed of statements randomly selected from the same pool as that used in the preceding conditions (Condition ROP).

\section{Procedure}

The experiment was conducted in a language laboratory. Each of the nine Ss worked in an isolated booth and wore headsets. Only the timing signals and instructions for all phases of the experiment were tape recorded and received by the $S$ as he read a printed version of the instructions included in an answer booklet. The language laboratory facility permitted the simultaneous presentation of instructions for nine different experimental conditions.

The S's task was to learn as much as possible from the passage assigned to him. Each of three study-recall trials consisted of a 3-min study period. during which time $S s$ were instructed to read and study the complete passage without the assistance of notes, and a 6 -min writing period. during which time they were instructed to write down all of the statements that could be recalled, in any order (i.e., the free-recall test).

\section{Organization Treatments}

One experimental variation consisted of manipulating the organization of the passage as described in the above section on experimental materials. Briefly, Ss were randomly assigned to study CNP, CAP, or ROP passages.

\section{Instructional Sets}

The Ss assigned to each of the passage organization treatments were further assigned on a random basis to one of three instructional treatments: One-third of the Ss were instructed to learn the passage by grouping the material according to the concept name (CNI), another third were to learn the passage by grouping the material by concept attribute (CAI), and the final third were instructed to use whatever plan or strategy they felt would best help them remember the passage (NOI).

The key sentence for these manipulations was as illustrated in the following instructions for the CNI treatment: "In this experiment we are interested in the strategies or plans students use to learn written material like that contained in the passage you will study. One strategy is to group similar statements together and then to remember them as a group. As you study this material, we want you to group all the statements about a given nation together and to learn them this way." This instruction was followed by two specific illustrations of the rule for organizing the three statements for each of two imaginary nations. The final statement in the set of instructions was, "Your task is to learn the statements in this passage by grouping them this way regardless of the way they are presented in the passage." The same general instructions were used for the CAl treatment. except for the phrase "... we want you to group all the statements about the same type of characteristic [attribute] together...." The illustrations. of course. paralleled the instruction. The instructions for the RNI treatment suggested several strategies that might be employed in organizing the material and concluded with the statement. "Your task is to learn the statements by using whatever plan or strateg. for remembering is easiest for you."

\section{Inten'ening Task}

Following the third trial, Ss were given a 10 -min short-term memory task (Peterson \& Peterson, 1959) designed to prevent rehearsal of the passage and to provide a measure of memory span. Immediately following this interval, a fourth free-recall test was administered but was not preceded by a study period.

\section{Association Test}

An association test followed the fourth writing period. For this task, 12 cue statements from the passage were read to the Ss. They were instructed to respond to each cue by writing the first statement from the paragraph that came to mind. For example, when the cue, "Egrama is characterized by a mountainous terrain." was read, the S could respond with another statement about Egrama (suggesting name organization) or with a statement about the geography of a different country (suggesting attribute organization). Since each cue statement contained a name and an attribute, the S's responses could be analyzed to determine the ratio of name to attribute associations.

A postexperimental questionnaire was administered at the conclusion of the experiment. The $S$ was asked to describe his strategy for learning the statements in the passage. He also rated the instructional treatment on a five-point scale consisting of the following designations: very helpful, somewhat helpful. neither helpful nor interfering, somewhat interfering, or very interfering.

\section{Scoring}

The free-recall protocols were scored according to procedures described more fully by Schultz and Di Vesta (1972). Briefly, the scores consisted of the number of correct statements recalled. name clustering ratios (which reflect the amount of organization in free recall by concept name), and attribute clustering ratios (which reflect the amount of organization in free recall by concept attribute). A sequence within clusters (SWC) score was also obtained to reflect the degree of consistency of organization across clusters within a given trial. It was used to indicate the extent to which the sequence of statements within the dominant bases for organization (i.e.. name or attribute) was in the same order from one cluster to the next.

\section{RESULTS}

The presentation of results is based on separate analyses of variance made of two clustering scores (name and attribute clusters) and statements correctly recalled. These analyses included free-recall trials. Additional analyses were made of association scores and the sequence within clusters (SWC) scores for the fourth trial only. Except where noted otherwise, Newman-Keuls procedures were used for all multiple comparisons. The means for all treatments. based on each dependent variable, are summarized in Table 1. 


\section{Induction of Treatments}

The association test was used to determine the extent to which the experimental conditions were induced. A score was derived by subtracting the attribute clustering ratio from the name clustering ratio and adding 1 to eliminate negative numbers (i.e., the range of scores was $0-2)$ : High scores reflect name organization and low scores reflect attribute organization.

An analysis of variance of these data yielded $F(2,90)=9.35, p<.01$ for the effect due to passage organization, in which the following order of means was obtained: $\overline{\mathrm{X}}=1.55$ for CNP, 1.26 for ROP, and .87 for CAP; ROP and CNP differed from CAP $(p<.05)$ but not from each other. The effect due to instructions yielded $F(2,90)=3.53, p<.05$. Mean scores for $\mathrm{CNI}$, NOI, and CAI were $1.47,1.08$, and 1.14 , respectively. The mean score for $\mathrm{CNI}$ was greater $(\mathrm{p}<.05)$ than those for CAI and NOI, which did not differ $(p>.05)$ from each. other. These results indicate that the type of organization (name or attribute) employed by the $S$ was influenced, or induced, respectively, by cues from both the passage organization and the instructions to employ different learning strategies.

\section{Effects of Organization and Instructions}

Since one purpose of this study was to identify the effects of the interaction between instructional sets and passage organization, it was necessary to determine whether results of previous studies were replicated. In this respect, the effects due to organization were significant $(p<.01)$ in analyses based on all scores. Organization influenced (a) the number of statements correctly recalled $[F(2,90)=5.94]$, (b) name clustering $[F(2,90)=6.66], \quad$ (c) attribute clustering $[F(2,90)=5.01]$, and (d) SWC $[F(2,90)=14.79]$. In general, Ss tended to organize material during recall in the same way as it was organized during presentation. Without organization, Ss recalled fewer statements than did Ss who received organized material, and they clustered their responses by concept-name rather than by concept-attribute categories (see Table 1).

All clustering scores were affected significantly $(p<.01)$ by instructions: $F(2,90)=35.20$ for name clustering, $F(2,90)=39.19$ for attribute clustering, and $F(2,90)=10.05$ for SWC. Thus, organization for information during retrieval is influenced by the strategy imposed, through instructional sets, for organizing the material during learning. In all analyses, the means of the clustering ratios for NOI were between those for the $\mathrm{CNI}$ and CAI treatments.

The triple interaction of Organization by Instructions by Trials for attribute clustering scores yielded $F(12,270)=2.08, p<.01$. An analysis of interaction contrasts indicated that the Trials by Instructions interaction was not significant $(p>.05)$ when either the CNP or ROP organizations were learned. However, this
Table 1

Summary of Mean Scores on Each Dependent Variable for Each of the Experimental Conditions

\begin{tabular}{ccccc} 
& \multicolumn{3}{c}{ Organization of Passage } \\
\cline { 2 - 3 } Instructions & $\begin{array}{c}\text { Random } \\
\text { (ROP) }\end{array}$ & $\begin{array}{c}\text { Attribute } \\
\text { (CAP) }\end{array}$ & $\begin{array}{c}\text { Name } \\
\text { (CNP) }\end{array}$ & Total \\
\hline Correct & Statements (SE of Mean $=1.39)$ & \\
None (ROI) & 11.41 & 12.07 & 11.57 & 11.68 \\
Attribute (CAI) & 9.82 & 12.41 & 17.48 & 13.23 \\
Name (CNI) & 9.80 & 14.91 & 13.25 & 12.65 \\
Total & 10.34 & 13.12 & 14.10 & \\
Name Clustering & Ratios & (SE of Mean $=8.40)$ & \\
None (ROI) & 41.91 & 42.18 & 64.52 & 49.54 \\
Attribute (CAI) & 29.52 & 13.45 & 41.93 & 28.30 \\
Name (CNI) & 65.45 & 90.61 & $99.63 *$ & 85.23 \\
Total & 45.63 & 48.75 & 68.70 & \\
Attribute Clustering Ratios & (SE of Mean $=7.89)$ \\
None (ROI) & 25.95 & 43.68 & 15.68 & 28.44 \\
Attribute (CAI) & 56.02 & 81.20 & 50.98 & 62.73 \\
Name (CNI) & 13.41 & 3.89 & $.98 * *$ & 6.09 \\
Total & 31.80 & 42.92 & 22.54 & \\
Intercluster Consistency (SCW) (SE of Mean $=7.85)$ \\
None (ROI) & 31.27 & 59.09 & 48.82 & 46.39 \\
Attribute (CAI) & 50.36 & 67.00 & $91.36 * * * 69.58$ \\
Name (CNI) & 47.91 & 81.45 & $89.23 \dagger$ & 72.70 \\
Total & 43.18 & 69.18 & 76.30 & \\
Ratings of & Instructions & (SE of Mean $=0.27)$ & \\
None (ROI) & 2.91 & $2.55+\dagger$ & $2.73 \dagger \dagger \dagger$ & 2.77 \\
Attribute (CAI) & 2.64 & 2.55 & 1.82 & 2.33 \\
Name (CNI) & 2.36 & 2.00 & 2.45 & 2.27 \\
Total & 2.63 & 2.36 & 2.33 & \\
\hline
\end{tabular}

Note-The hypothesis of homogeneity of variance was not tenable for the sets of means in which footnotes appear. For the significant departures, the $S E$ of mean values were as follow: $* 0.26,{ }^{* *} 0.67,{ }^{* *} 4.95,+3.01,++0.16$, and +++0.14 .

interaction was significant for learning materials with CAP organization $[F(6,90)=3.67, \mathrm{p}<.05]$, i.e., the CAI group adopted the attribute clustering strategy immediately, whereas it was not used in the CNI group.

The attribute clustering ratios for the NOI group increased linearly from the first to fourth trials. On the first trial, the mean score for CAI was significantly higher than the means for either NOI and $\mathrm{CNI}$, which did not differ from each other $(p<.05)$. On the fourth trial, the mean attribute clustering ratio for $\mathrm{CAI}$ and NOI did not differ from each other but both were significantly $(p<.05)$ higher than that for CNI. This finding clearly supports the hypothesis that the adoption of a subordinate clustering strategy is incremental.

In contrast, the concept-name clustering strategy was adopted on the first trial when no instructional sets were provided. A contrast to test the significance of the interaction of Name and Attribute Passage Organization by Trial 1 and Trial 4 for the NOI group yielded $t=2.67, p<.01$.

Remembering that high scores reflect name organization while low scores reflect attribute 


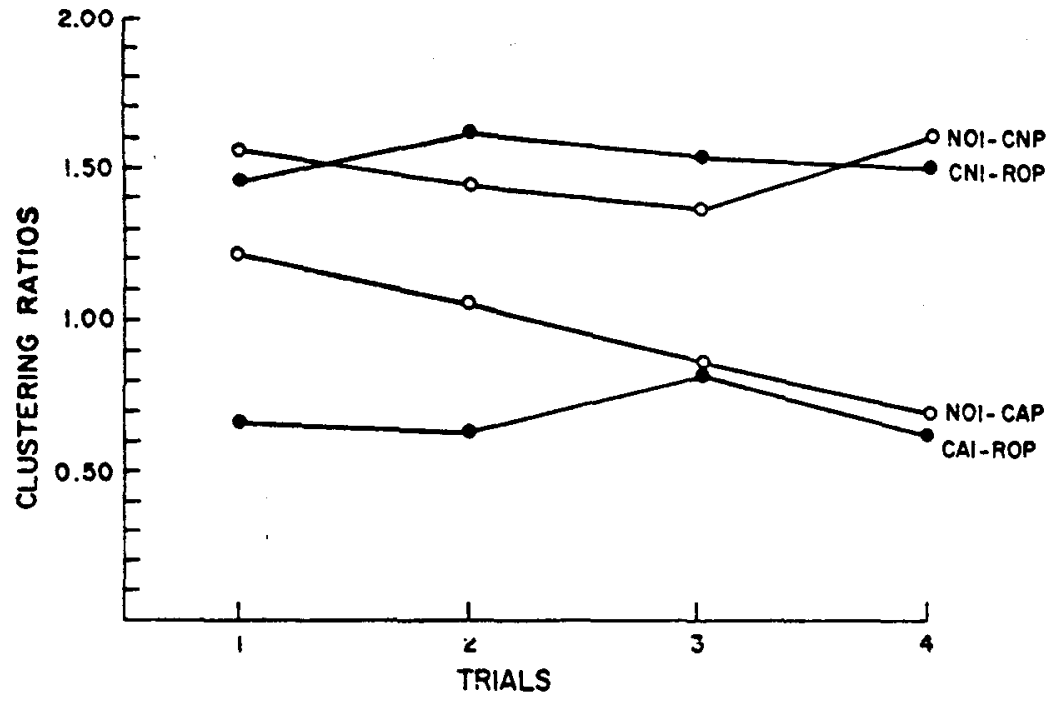

Fig. 1. Clustering scores based on differences between name clustering scores and attribute clustering scores for four instruction and organization groups across trials.

organization, the means for the above analyses are graphically displayed in Fig. 1. There it may be seen that the strategy of organizing by name (Group NOI-CNP) was immediately adopted and maintained, while the adoption of the strategy of organizing by attribute (Group NOI-CAP) was incremental over trials. By Trial 4, the NOI-CAP group differed significantly from the NOI-CNP group $[\mathrm{t}(90)=2.05, \mathrm{p}<.05]$.

In order to determine whether one strategy was generally more dominant than the other, a $t$ test of correlated means of the name and attribute clustering ratios was made. This analysis yielded $t(98)=3.11$, $\mathrm{p}<.01$, implying preference for name over attribute strategies, thereby providing direct support for earlier findings by Frase (1969) and Schultz and Di Vesta (1972).

\section{Effect of Congruency Between Organization and Instructions}

The primary interest in the present study was the comparison of recall by $\mathrm{Ss}$ who received passage organization either congruent or incongruent with instructions on how to organize the material during learning. Initially, it was hypothesized that congruence between passage organization and instructions would result in greater recall than incongruence.

The dependent variable for the analyses implied by this hypothesis was the number of statements correctly recalled. This analysis yielded $F<1.0$ for the main effect due to instructions. A similar analysis of the effect due to passage organization yielded $F(2,90)=5.94$, $\mathrm{p}<.01$, indicating that both the CNP and CAP groups recalled more than the ROP group but did not differ from each other. The analysis also yielded a significant effect $[F(3,270)=204.51, p<.01]$ due to trials and a significant Trials by Organization interaction $[F(6,270)=2.52, p<.05]$. The most important feature of this interaction is the tendency for the superiority of recall in the CNP and CAP groups relative to that of the
ROP group to be increasingly marked with additional trials until, by the fourth trial, the groups receiving organized passages were significantly higher than the groups receiving the unorganized passages.

The result specific to the congruency hypothesis was the significant interaction of Organization by Instruction $[F(4,90)=2.78, p<.05]$. The means are summarized in Table 1 under the heading of Correct Statements. A comparison of simple effects with the Newman-Keuls test yielded the following: (a) recall by the NOI group did not differ significantly $(p>.05)$ across different passage organization treatments; (b) Ss in the CNI groups recalled significantly $(p<.05)$ more from both the CNP and CAP passages than from the ROP passage; (c) the CAI group recalled significantly $(p<.05)$ more from the CNP passage than from the CAP or ROP passages; and (d) the CAI group recalled significantly $(\mathrm{p}<.05)$ more than the $\mathrm{CNI}$ and NOI groups from the CNP passage.

As can be seen in (c) and (d) above, the results contradict the hypothesized direction: Scores for groups in which both manipulations were congruent (i.e., attribute organization with attribute instructions and name organization with name instructions) were lower than scores for groups in which both manipulations were incongruent (i.e., attribute organization with name instructions and name organization with attribute instructions). A specific test involving the four means within this interaction, and implied by the direction of the means, yielded $t(90)=2.43, p<.02$, indicating significantly greater recall when the passage organization and instructions (learning strategies) were incongruent than when they were congruent. (See the corresponding cell means for both $\mathrm{CN}$ and $\mathrm{CA}$ groups in Table 1.)

The subjective reports provided an independent measure of the outcomes of the experimental treatments inasmuch as the Ss received no evaluational feedback, neither norm nor criterion referenced, about their performance. Again, the analysis of particular interest involves the subjective reports of $\mathrm{Ss}$ assigned to the cells 
represented by the interaction of Name or Attribute Instructional Sets by Name or Attribute Passage Organization. The interaction contrast involving these four cells yielded $t(90)=2.18, p<.05$. As may be seen in Table 1, the magnitude of these ratings varied directly with the magnitude of the recall scores. Thus, when passage organization and instructional set were incongruent, the instructions were rated as more helpful (represented by lower scores) than when the task requirements and passage organization were congruent.

\section{DISCUSSION}

The results of this study clearly support the hypothesis that both the organization of the passage and instructions influence the amount of material recalled from the meaningful passages and the manner in which learners organize material during recall. The fact that more ideas are recalled when subsumed within an organizational scheme is compatible with the assumption that organization is an important component of cognitive structure (Ausubel, 1968). Clear support was also provided for the hypothesis that Ss initially adopt a name clustering strategy as a dominant strategy and gradually relinquish it for the attribute strategy when required to do so.

Parenthetically, it should be noted that Myers et al (1972) found attribute organization to result in superior recall over name organization. They explained their results as follows: "It appears that the superior recall of A [attribute] subjects is primarily due to the presence of a serial-order retrieval scheme which is natural and easy to use, and quite effective [p. 14]." However, there were many differences (including use of quite different materials and both free- and serial-recall procedures) between the Myers et al study, the earlier ones, and the present one. Thus, it is impossible to determine the exact locus of the superiority of attribute passage organization in the Myers et al study. Nevertheless, together with the earlier studies and the present one, the Myers et al study implies the need for more analytic studies of attribute and name passage organization in future investigations. For example, none of these studies has investigated the effect of organization on long-term vs short-term recall.

The findings regarding the congruency-incongruency hypothesis in the present study can be explained tentatively in terms of Restle's (1962) model. He contends that difficulties in cue learning are encountered when Ss' learning strategies conflict with the strategies intended by the E. Such situations are experienced by learners when the passage is organized according to attribute and no instructions are administered other than those to memorize the material for later recall. Difficulty in learning may be experienced because the dominant concept-name strategy must be abandoned to be replaced by a subordinate one. Accordingly, the learning curve, based on performance of the group that must learn the passage organized by attributes (CAP-NOI), reflects a gradual change from the strategy or organizing by name to the strategy of organizing by attribute.

The gradual adoption of a new strategy, where its use is not clear to the learner, differs radically from the rather abrupt and spontaneous adoption by learners instructed to use an appropriate, relevant, or salient strategy. The statement by Johnson, Fishkin, and Bourne (1966) that "... instructions which include explicit labels for stimulus dimensions, which indicate only one dimension will be relevant and which demonstrate a possible solution to a problem all combine to induce a hypothesis-testing type of situation [p. 70]" seems as appropriate to the adoption of a strategy for organizing connected discourse as it is to concept learning.

The main thesis of Restle's (1962) model is supported in this study by the effect of the interaction between passage organization (i.e., name and attribute only) and instructional treatment (i.e., name and attribute only) on recall, where incongruency between the two was more facilitative than congruency. It will be recalled that the Ss rated the incongruent conditions as more helpful than the conditions in which the instructional sets coincided with passage organization. Thus, incongruent instructions appear to define, for the learner, the two fundamental rules for organizing the material to be learned: One of these rules is obtained more or less implicitly from the passage organization; the other is obtained explicitly from the instructional set. By employing these two rules, the learner can approximate a matrix that would eventually permit him to make application (transfer) to other situations.

The aforegoing conclusion is parallel with one by Hagen, Meacham, and Mesibov (1970) that "Verbal labels which are imposed externally are irrelevant, and even distracting for the individual who does not utilize them for task performance [p. 57]." However, when materials are organized by concept name, instructions to use the concept-attribute organizing strategy provides supplementary information, i.e., the combination informs the learner that the material can be organized in at least two ways. A similar situation exists for the CAP-CNI group. Thus, in the incongruent conditions the two rules are given: one implied in the organization and the other made explicit by instructions. Consistent with Hagen, Meacham, and Mesibov's (1970) comment, instructions, even though ostensibly incongruent with passage organization, need not be irrelevant unless they cannot be or are chosen not to be used by the learner when performing the task. The data imply that, in the present experiment, the learner did use these rules. Moreover, (s)he rated them as helpful. Upon post hoc reasoning, the explanation appears to be that incongruency between instructions and passage organization in a task involving only two strategies is more informative than a congruent one that taps only a 
single strategy. In such situations, incongruency exhausts the space scanned by the learner, thereby limiting the amount of search or discovery needed for classification of ideas. As a consequence, his learning is more efficient than it would be otherwise.

\section{REFERENCES}

Ausubel, D. P. Educational psychology: A cognitive vien: New York: Holt, Rinehart. \& Winston. 1968.

Frase, L. T. Paragraph organization of written materials: The influence of conceptual clustering upon the level and organization of recall. Journal of Educational Psychology, $1969,60,394-401$.

Hagen, J. W., Meacham, J. A.. \& Mesibov, G. Verbal labeling, rehearsal. and short-term memory. Cognitive Psychology. $1970,1.47-58$.

Johnson, P. J.. Fishkin. A.. \& Bourne, L. E., Jr. Effects of procedural variables on reversal and interdimensional shift performance: Part II. Psychonomic Science, 1966, 4. 69-70. Part I. 63-64.

Myers, J. L., Pezdek, K., \& Coulson, D. Effects of prose organization upon free recall. Report 72-1. Amherst, Mass: Cognitive Processes Laboratory, University of Massachusetts. 1972.

Peterson. L. R., \& Peterson. M. J. Short-term retention of individual items. Journal of Experimental Psychology, 1959, 58. 193-198.

Restle, F. The selection of strategies in cue learning. Psychological Review, 1962, 69, 329-343.

Schultz, C. B.. \& Di Vesta, F. J. The effects of passage organization and note-taking on the selection of clustering strategies and on recall of textual materials. Journal of Educational Psychology. 1972, 63, 244-252.

(Received for publication January 15, 1973; revision received April 2, 1973; accepted April 19, 1973.) 